\title{
Obesidade, depressão e estresse: relato de uma intervenção multidisciplinar em grupo com adolescentes
}

\section{obesity, depression and stress: a report about a multidisciplinary intervention with a group of adolescents}

\author{
Lucas Lüdtke ${ }^{1}$ Bárbara Maria Barbosa Silva², Ana Carolina Wolf Baldino Peuker², Edna \\ Linhares Garcia ${ }^{1,3}$ \\ 1 - Universidade de Santa Cruz do Sul - UNISC, Santa Cruz do Sul, RS, Brasil. \\ 2 - Universidade Federal do Rio Grande do Sul - UFRGS, Porto Alegre, RS, Brasil. \\ 3 - Universidade Católica de São Paulo - PUC/SP, São Paulo, SP, Brasil.
}

lucasludtke@hotmail.com

Estresse; Estudo

Comparativo.
Palavras-chave: Obesidade; Depressão;

\section{RESUMO}

Objetivo: o presente estudo é do tipo quase-experimental e tem como objetivo avaliar a relação entre obesidade, depressão e estresse. Método: trata-se de uma amostra de 46 escolares da cidade de Santa Cruz do Sul - RS, com idades entre 11 e 17 anos e de ambos os sexos, que foram divididos em grupo controle (GC) e grupo experimental (GE). O GE participou de um programa de intervenção no período de 3 meses que englobou as áreas de Educação Física, Nutrição e Psicologia. O GE participou de um programa previamente estruturado de 13 sessões, no formato de grupo, do tipo orientação e treinamento cognitivo-comportamental. Para avaliar a presença e a fase do estresse foi utilizada a Escala de Stress Infantil e a Escala de Stress para Adolescentes. Para avaliar a presença de sintomas depressivos e sua intensidade, foi utilizado o Inventário de Depressão de Beck (BDI-II). Resultados: não foram encontrados índices significativos de estresse no comparativo dos grupos. A amostra apresentou níveis de significância em relação à intensidade do quadro depressivo, reduzindo nos comparativos de pré e pós teste. Os resultados do presente estudo podem ser observados conforme os Índices de Massa Corporal (IMC) comparados através do teste "t student" entre GC e GE. Para o GE, observa-se uma redução média do IMC de $\mathrm{M}=-1,21 \mathrm{~kg} / \mathrm{m}^{2} / \mathrm{DP}=2,26$, aproximadamente o dobro quanto ao GC uma redução de $\mathrm{M}=-0,63 \mathrm{~kg} / \mathrm{m}^{2} / \mathrm{DP}=1,42$; em relação ao peso, o $\mathrm{GE}$ apresentou redução de $\mathrm{M}=-1,19 \mathrm{~kg}$ / $\mathrm{DP}=4,94$ e o $\mathrm{GC} \mathrm{M}=-0,71 \mathrm{~kg} / \mathrm{DP}=3,56$. Estes dados mostram uma redução significativa no IMC e na perda de peso do GE quando comparados ao GC. Conclusão: houve eficiência na intervenção quanto à perda de peso, redução de sintomas depressivos e a mudança cognitiva, com aquisição de novas habilidades mentais pelo GE.

\section{ABSTRACT}

Objective: this was a quasi-experimental study that aimed to evaluate the relationship among obesity, depression and stress. Method: a sample of 46 schools was selected in the city of Santa Cruz do Sul-Rio Grande do Sul, with male and female students between the ages of 11 and 17 years, which were divided into a control group (CG) and an experimental group (EG). The EG participated in a three-month intervention program that covered the areas of physical education, nutrition and psychology. The EG participated in a program structured in 13 group counseling and cognitive-behavioral training sessions. To evaluate the presence and phase of stress, the Infant Stress scale and the Adolescent Stress scale were employed. The presence and intensity of depressive symptoms were measured using the Beck Depression Inventory (BDI-II). Results: no significant differences were found in the stress indexes between the groups. The intensity of depression significantly decreased from pre to post intervention. The results of the body mass index (BMI) between the CG and EG were compared using Student's t test. The EG, showed a mean reduction in BMI of $\mathrm{M}=-1.21 \mathrm{~kg} / \mathrm{m}^{2} / \mathrm{PD}=2.26$, approximately twice as much for the $\mathrm{CG}$, with a reduction of $\mathrm{M}=-0.63 \mathrm{~kg} / \mathrm{m}^{2} / \mathrm{PD}=1.42$. In terms of weight, the EG presented a reduction of $\mathrm{M}=-1.19 \mathrm{~kg} / \mathrm{PD}=4.94$ and the $\mathrm{CG}$, of $\mathrm{M}=-0.71 \mathrm{~kg} / \mathrm{PD}$

Keywords: $=3.56$. These data show a significant reduction in BMI and weight loss among the EG when compared Obesity; Depression; to the CG. Conclusion: the intervention proved to be effective in terms of weight loss, reduction of Stress; Comparative study. depressive symptoms and cognitive change, with the EG demonstrating new mental abilities by. 


\section{INTRODUÇÃO}

A obesidade, nas últimas décadas, vem atingindo proporções grandiosas em vários países, podendo ser considerada um problema de saúde pública mundial. É uma condição grave e de muitas causas, dentre as quais se encontram fatores genéticos, culturais e do desenvolvimento psicológico e familiar. É uma condição multicausal que se manifesta em diversas faixas etárias. A obesidade não é considerada um transtorno mental, é o resultado do excesso prolongado de ingestão energética em relação ao gasto energético, portanto possuir obesidade não significa presença de transtornos psicológicos. ${ }^{1-4}$

Segundo Godoy-Matos et al., ${ }^{2}$ a obesidade, do ponto de vista psicológico, não pode ser mais considerada meramente o resultado de uma manifestação somática de um conflito psicológico subjacente, como se acreditou por muito tempo. Do ponto de vista fisiológico, a obesidade pode ser considerada um acúmulo anormal de gordura no organismo e que leva a algum comprometimento da saúde. Quando há um desequilíbrio entre a ingestão e o gasto, ocorre um armazenamento excessivo ou anormal de energia no tecido adiposo e isto levará à obesidade. Para a psicologia cognitivocomportamental, é importante compreender quais são os fatores psicológicos envolvidos neste desequilíbrio entre a ingesta e gasto calórico e quais destes aspectos psicológicos contribuem ou não para levar os indivíduos à obesidade. $\mathrm{O}$ presente estudo procurou avaliar a forma de pensar dos participantes em relação a esta condição, visando atuar tanto nos contribuintes ou consequentes relacionados à obesidade, levando à diminuição do sofrimento psicológico e conduzindo a uma melhor condição de saúde mental. ${ }^{5-7}$

Em torno de 1\% dos casos de obesidade são de causa genética ou endócrina e os $99 \%$ restantes constituem causas externas. A obesidade é considerada um fenômeno no qual a cognição, comportamento e emoções irão interagir mutuamente, sendo influenciada por padrões nutricionais e atividade física, entre outros. ${ }^{1,8}$ Por outro lado, alguns estudos consideram necessária uma abordagem multidisciplinar e interdisciplinar do problema, pois seu tratamento leva profissionais e pacientes constantemente a sentiremse frustrados..$^{9,10} \quad$ Finger, Potter ${ }^{11}$ mostram que, no Brasil, há prevalência de $20,9 \%$ de jovens com sobrepeso e $5 \%$ com obesidade. Em adultos brasileiros, se encontram as seguintes taxas: $47 \%$ dos homens e
$39 \%$ das mulheres com sobrepeso e 11\% para ambos com obesidade. Já, para crianças de dois a seis anos de idade. aponta Gonçalves, Silva, Antunes, ${ }^{5}$, há prevalência de sobrepeso e obesidade de $37,2 \%$ para o sexo masculino e $33,4 \%$ para o sexo feminino. Segundo Luiz Gorayeb, Liberatorre Júnior, ${ }^{12} 24,8 \%$ de escolares entre 11 a 13 anos apresentam sobrepeso e obesidade no Rio Grande do Sul. Muitos adolescentes obesos sofrem constantemente preconceito, o que interfere no seu funcionamento psicológico e gera um impacto negativo em suas vidas, tornando sua aceitação social e percepção de si mesmo negativa. Dessa forma, adolescentes que são obesos passam por muitos problemas em relação à aceitação de sua autoimagem e à valorização de seu próprio corpo. Muitas vezes, estes não acreditam ter condições de mudar suas atitudes quanto ao comer e não aderem a tratamentos. Tal desregulação pode ser considerada por aspectos emocionais e pode gerar um ciclo vicioso de tensão-comer-mais/tensão-mais comer. ${ }^{5,10-14}$

Dentre os aspectos psicológicos mais comuns em adolescentes obesos apontados nos estudos pesquisados estão à infantilização, vitimização, percepção distorcida de si mesmo, dificuldades no desenvolvimento emocional, dificuldades comportamentais, dificuldade de adiar satisfações, dificuldade em lidar com frustrações, dificuldade em obter prazer nas relações sociais, dificuldade em lidar com a sexualidade, falta de limites, dependência materna, baixa autoestima, fraca interação social, dificuldade no controle de impulsos, sintomas depressivos, não aceitação da aparência física, carência afetiva, insatisfação, desânimo, sentimento de inferioridade, sintomas ansiosos, angústia, frustração, tensão, estresse, apatia, insegurança, raiva, tristeza, imaturidade e passividade. , $^{1,5,9,10,12-18}$

Embora estudos correlacionem aspectos psicológicos como causa ou consequência da obesidade, os autores Gonçalves, Silva, Antunes ${ }^{5}$, Sarmento, Schoen-Ferreira, Medeiros, Cintra ${ }^{13}$, Cataneo, Carvalho, Galindo ${ }^{14}$ e Luiz, Gorayeb, Liberatore Júnior, Domingos ${ }^{19}$ demonstram não existir diferença significativa entre adolescentes obesos e não-obesos e salientam as pesquisas existentes como modestas e nem sempre conclusivas, pois ser obeso não necessariamente implicará em transtornos psicológicos.

Conforme as Diretrizes Brasileiras de Obesidade - ABESO, a Terapia CognitivoComportamental em Grupo (TCCG) é considerada 
um importante auxiliar no tratamento da obesidade. Com o desenvolvimento da Terapia CognitivoComportamental (TCC), vários pesquisadores têm se voltado a estudar essa abordagem em grupos. Os pressupostos teóricos adotados em grupo são os mesmos utilizados individualmente. A TCCG possui um sistema de funcionamento que possibilita o uso de técnicas específicas com objetivo a atingir resultados específicos na redução de problemas psíquicos. Esta leva em consideração a homogeneidade na composição dos seus grupos, tendo estes metas e características comuns, e é o que vai diferenciar esta modalidade das demais modalidades grupais. Os critérios de inclusão e exclusão dos seus participantes devem se dar de forma clara e com demandas específicas. A TCC em grupo parte do princípio que os indivíduos processam informações, recebendo dados e gerando a apreciação destes. Utiliza dos princípios teóricos da aprendizagem social onde são estimados a cultura, valores, significados, crenças e expectativas dos indivíduos. ${ }^{2,20-22}$

Programas de prevenção e promoção no campo da saúde podem ser considerados assuntos novos para a psicologia. Estes possuem semelhanças significativas entre si, mas a principal diferença reside nas metas de cada um. As ações preventivas buscam reduzir a incidência e a prevalência de doenças sendo orientadas a evitar o seu surgimento. As ações de promoção buscam fortalecer os indivíduos de forma coletiva ou individual a lidar com os condicionantes da saúde, promovendo assim o bem estar de forma global e não focando apenas em uma doença ou transtorno. ${ }^{20,23}$

O tratamento psicológico para o fenômeno da obesidade envolve uma melhora na qualidade de vida dos indivíduos por ela acometidos. Implica em oportunizar iniciativas que auxiliem na conquista de uma melhora na saúde mental, focalizando seus esforços para a diminuição do sofrimento e aumento do bem-estar cognitivo, não tratando meramente sinais ou sintomas da obesidade. ${ }^{1,3,20}$

A TCC pressupõe que o pensamento distorcido ou disfuncional afeta o humor e o comportamento dos indivíduos. Uma avaliação mais realista e a modificação destes pensamentos mantêm a melhora dos comportamentos e do humor, facilitando formas mais adaptativas e saudáveis de funcionar. Promover saúde significa ampliar a consciência que os indivíduos têm sobre a própria realidade, instrumentando-o para agir, no sentido de mudar e resolver as dificuldades que essa realidade lhe apresenta. ${ }^{20,24}$

No que se refere em relação às características de intervenção Pereira e Silva ${ }^{25}$ sugerem que os efeitos dos programas na obesidade devem considerar a duração, o conteúdo informativo, dieta e exercícios físicos. Os estudos interventivos da psicologia na obesidade de Apienza, Schoen, Fisberg, ${ }^{26}$ Neufeld, Moreira, Xavier ${ }^{21}$ e Pereira ${ }^{27}$ apresentam resultados positivos de seus programas de intervenção no que diz respeito ao IMC, porém com resultados um pouco contraditórios nos aspectos, sinais e sintomas psicológicos na obesidade, pois não utilizam instrumentos de medida ou testes psicológicos para tal.

Por não existir um consenso nos estudos analisados sobre a associação de aspectos psicológicos relacionados à obesidade, tanto como antecedentes ou consequentes, o presente estudo objetivou avaliar a possível relação de sintomas depressivos e estresse em uma amostra de escolares com sobrepeso e obesidade. O trabalho também objetiva descrever a intervenção estruturada que foi realizada no grupo experimental de forma mais detalhada pela Psicologia.

\section{PARTICIPANTES E PROCEDIMENTOS}

O presente estudo é do tipo quaseexperimental e integra um programa mais amplo denominado "Obesidade em escolares da educação básica: um estudo de intervenção interdisciplinar", aprovado pelo Comitê de Ética em Pesquisa (CEP) da Universidade de Santa Cruz do Sul (UNISC) sob o protocolo $n^{\circ} 357.403$. As intervenções ocorreram de forma interdisciplinar e englobaram as áreas de Educação Física, Nutrição, Fisioterapia, Psicologia e Odontologia. As intervenções aconteceram durante três dias da semana (segunda, quarta e sexta) pelo período de três meses no Grupo Experimental (GE), de maio a julho do ano de 2015. O programa inclui principalmente exercícios físicos nos três dias da semana orientações com psicólogo e nutricionista uma vez por semana. As coletas do Grupo Controle (GC) ocorreram no mês de setembro de 2015 e no mês de novembro de 2015. Neste trabalho, direcionase o foco para a intervenção realizada pela psicologia, objetivando relatar a experiência resultante de uma intervenção da Terapia Cognitivo-Comportamental em Grupo (TCCG), modalidade grupo de orientação e treinamento cognitivo para escolares com sobrepeso e obesidade. O programa de sessões constituiu-se de 
13 encontros de orientação cognitiva, sendo cada um com duração média de 50 minutos para o GE. O GC participou apenas das coletas dos testes, não passando por interevenção de nenhuma área.

A amostra foi constituída por 46 escolares com sobrepeso e obesidade com idade compreendida entre 11 a 17 anos, de ambos os sexos, selecionados voluntariamente em 25 escolas do município de Santa Cruz do Sul - RS. Tal amostra foi selecionada a partir de uma pesquisa transversal chamada "Saúde dos escolares - Fase III", que avaliou 25 escolas do município de 2014 a 2015. As escolas foram selecionadas por critério de conveniência, compreendendo escolas próximas à UNISC. Para que não houvesse interferência do programa de intervenção no estilo de vida do GC, os escolares alocados em GE e GC eram provenientes de escolas diferentes, mas com características semelhantes. Foram convidados 172 alunos para participar do programa, provenientes de sete escolas.

Foi realizado teste "t de student" em comparação aos dois grupos, para testar as diferenças e semelhanças entre os grupos, não ocorrendo diferenças significativas entre eles nas avaliações préintervenção $(p>0,05)$. Estas foram selecionadas para integrar o programa de intervenção e foram divididas aleatoriamente em GC e GE. Destas escolas, foram convidados os alunos que, durante as avaliações, foram diagnosticados com sobrepeso e obesidade para participar do estudo, através do Índice de Massa Corporal (IMC). Ao final da seleção, 49 escolares aceitaram participar do programa de intervenção e obtiveram autorização dos seus pais/responsáveis através da assinatura do termo de consentimento livre e esclarecido. Para completar o GE, foi realizada divulgação via rádio, jornal e internet e junto a quatro escolas, quando mais cinco sujeitos aceitaram aderir ao programa, totalizando 54 escolares inscritos para a intervenção. Entre o início do programa e o aceite de participação ocorreram 5 perdas no grupo de intervenção, pelo fato destes não possuírem autorização dos pais. O GE iniciou as intervenções com 49 participantes e finalizou com 23 , com uma perda total de 26 membros que não aderiram ao programa, em sua maioria por possuírem um grande número de faltas, sendo estes desligados do programa. O GC foi constituído de 23 membros.

Obedeceram-se os seguintes critérios de inclusão para a participação no estudo: a) aluno devidamente matriculado na escola selecionada que tenha aceitado participar da pesquisa; b) o responsável ter assinado o termo de consentimento livre e esclarecido; c) ter IMC maior que percentil 85 ; d) estar na faixa etária entre 10 a 17 anos; e) ter disponibilidade para frequentar o programa nos dias e horários determinados; f) nenhuma distinção em relação à classe, grupo social ou cor; g) ser de ambos os sexos; h) possuir obesidade exógena. Os critérios de exclusão foram: a) alunos que não preencherem os requisitos acima mencionados; b) que apresentaram uma frequência inferior a $80 \%$ de participação na intervenção ou apresentaram contraindicações, tais como retardo mental, transtornos psiquiátricos crônicos, transtornos alimentares, transtornos invasivos do desenvolvimento ou atraso do desenvolvimento cognitivo, que pudessem prejudicar a compreensão de instruções e estar em acompanhamento psicoterápico. Este estudo ofereceu risco mínimo aos participantes, conforme Resolução do Conselho Federal de Psicologia n. 016/2000. Os procedimentos utilizados nesta pesquisa também obedeceram aos Critérios da Ética na Pesquisa com Seres Humanos, conforme a Resolução no 466/12 do Conselho Nacional de Saúde.

\section{INTERVENÇÃO}

\section{Intervenção Nutricional}

A intervenção nutricional teve como foco principal a redução do consumo de açúcares, gorduras e sódio. Os encontros forram semanais, todas às sextas feiras, com duração de uma hora. Não foram prescritas dietas individuais. Os participantes foram acompanhados por nutricionistas. As atividades tiveram cunho educativa em relação ao conhecimento de vários tipos de alimentos e seus riscos à saúde, com o intuito de substituí-los e ou reduzir as porções nas ingestas diárias. Foram abordados temas como: visualização e identificação dos teores de sódio, açúcares e gorduras nos alimentos; riscos do consumo exagerado destes alimentos à saúde; rótulos nutricionais; quais as variedades de açúcares e gorduras presentes nos alimentos; indicação de produtos dietéticos conforme indicação de uso; opção de lanches mais saudáveis; identificação das porções diárias dos grupos de alimentos dentro da necessidade nutricional da faixa etária e a importância de hábitos como beber água, fracionar as refeições e a realização do café da manhã. 


\section{Intervenção com Exercícios Físicos}

As intervenções com exercícios físicos foram desenvolvidas por profissionais e bolsistas de Educação Física, ocorrendo três sessões semanais previamente programadas, que foram estruturadas da seguinte forma: às segundas-feiras, os escolares participavam de sessões desportivas que consistiam em aquecimento, alongamentos, processos pedagógicos do desporto e jogo; nas quartas-feiras, havia caminhadas seguidas por um circuito de exercícios resistidos/localizados; e nas sextas-feiras eram realizadas sessões de atividades na piscina do complexo esportivo com recreação, hidroginástica e iniciação ao nadar. Os treinos eram monitorados conforme a intensidade da frequência cardíaca (FC) máxima de cada participante entre $50 \%$ e $70 \%$. Os escolares controlavam a frequência através do monitor cardíaco portátil Polar FT1 (Polar, Finlândia) durante as sessões.

\section{Intervenção Psicológica}

Em relação à intervenção da psicologia no GE, esta consistiu em um grupo de terapia cognitivo-comportamental modalidade de orientação e treinamento para escolares com sobrepeso e obesidade. As intervenções ocorreram todas as segundas-feiras, com acompanhamento de psicólogo, totalizando 13 sessões de orientação cognitiva, tendo cada sessão uma duração média de 50 minutos para o grupo experimental. O programa de sessões está expresso na Tabela 1.

Descrição das técnicas utilizadas no programa de 13 sessões

As técnicas utilizadas no programa de 13 sessões no GE são descritas a seguir: "Estabelecimento de metas e objetivos"; 28,29 "Psicoeducação sobre o modelo da terapia cognitivo-comportamental"; ${ }^{28}$ "Psicoeducação sobre Pensamentos Automáticos - Influência do pensamento sobre sentimentos e comportamentos"; ${ }^{24,28-31}$ "Círculo mágico";24 "Psicoeducação sobre Pensamentos Automáticos Disfuncionais"; 24,28-31 "Perguntas que auxiliam na identificação de Pensamentos Automáticos Disfuncionais";32 "Registro de Pensamentos Disfuncionais";24,33 "Orientação sobre alimentação saudável"; 34 "Prática de exercícios físicos: Trabalhe as desculpas mais comuns para não praticar exercícios"; 34 "Distorções Cognitivas"; 30 "Mudando a maneira de pensar"; "Controlando estímulos que levam aos hábitos alimentares inadequados"; ${ }^{4}$ "Respiração diafragmática"; "Estratégia ACALME-SE"; 3 "Técnica Duplo-padrão";33 "Técnica Construção de Alternativas"; 33 "Técnica Observação da forma como os outros lidam com as coisas". ${ }^{33}$

\section{Instrumentos}

Para avaliar o estresse dos participantes foram utilizadas duas escalas, aplicadas conforme a faixa etária dos mesmos, a Escala de Stress Infantil (ESI) faixa etária: de 6 a 14 anos, de Lipp, Lucarelli, ${ }^{36}$ e a Escala de Stress para Adolescentes (ESA) faixa etária dos 14 aos 18 anos, de Tricoli, Lipp. ${ }^{37}$ As escalas foram aplicadas em grupo pelo psicólogo com dois auxiliares. Foram lidas as instruções e sanadas as dúvidas antes da aplicação. Durante a aplicação, alguns participantes foram auxiliados pelo profissional para maiores esclarecimentos. As duas escalas ESI e ESA permitiram avaliar e diagnosticar se os participantes possuem estresse e em qual fase se encontram, como alerta, resistência, quase-exaustão e exaustão. Tais escalas também permitem avaliar de forma específica a sintomatologia do estresse. A presente pesquisa deteve-se apenas em categorizar se os participantes apresentavam ou não estresse e em qual fase se encontravam, apesar de serem usadas as duas escalas elas aprensentam os mesmos resultados classificatórios.

Para avaliar os sintomas de depressão, foi utilizado o Inventário de Depressão de Beck (BDIII) de Gorenstein, Wang, Argimon, Werlang, ${ }^{38}$ versão revista, traduzida e adaptada para o português brasileiro.

O instrumento foi aplicado em grupo pelo psicólogo com dois auxiliares. $\mathrm{O}$ instrumento se aplica à faixa etária do presente estudo. Foram lidas as instruções e sanadas as dúvidas antes da aplicação. Durante a aplicação, alguns participantes foram auxiliados pelo profissional para maiores esclarecimentos. O BDIII consiste numa escala de auto-relato, composta por 21 conjuntos de declarações sobre os sintomas depressivos nos últimos 15 dias. Cada grupo apresenta quatro alternativas, que podem ser classificadas em uma escala ordinal de 0 a 3 , produzindo escores totais que variam de 0 a 63 , sendo subdivididos conforme a gravidade dos sintomas em: 0 a 13=mínimo; de 14 a $19=$ leve; de 20 a $28=$ moderado; e de 29 a 63 = grave. As questões presentes no BDI-II referemse à: 1) tristeza; 2) pessimismo; 3) fracasso passado; 4) perda de prazer; 5) sentimentos de culpa; 6) sentimentos de punição; 7) autoestima; 8) autocrítica; 
Tabela 1- Descrição da estrutura das sessões em grupo para orientação e treinamento cognitivo - grupo experimental

Sessão Objetivos da sessão

\section{Coleta de dados}

1

Apresentação do programa de sessões

Explicar o funcionamento e estrutura das sessões

Estruturação e psicoeducação sobre o modelo da terapia cognitiva

Estabelecer metas e objetivos

Revisão da tarefa de casa Orientação sobre alimentação saudável e a prática de exercícios físicos

Revisão da tarefa de casa Reestruturação cognitiva reconhecendo as distorções cognitivas

Revisão da tarefa de casa Estabelecer novas formas de avaliar as situações alimentares

Revisão da tarefa de casa Como controlar estímulos

Revisão da tarefa de casa Técnicas de relaxamento Revisão da tarefa de casa Psicoeducação em relação da diferença de fome - necessidade saciedade alimentar Estrạtégia ACALME-SE Revisão das tecnicas de relaxamento Trabalhar novamente o registro de pensamentos disfuncionais Aplicação do exercício duplo-padrão

Revisão da tarefa de casa Trabalho com técnicas cognitivas

Revisão da tarefa de casa Revisão de todas técnicas Feedback

13

Discussão sobre o programa Encerramento
Procedimentos

Tarefas para casa
Aplicação de instrumentos

Avaliação do IMC

Apresentação pessoal

Explicação do programa

Elaboração do contrato terapêutico

Estrutura das sessões - "miniaula", exercícios,

elaboração da tarefa de casa, revisão, feedback e

encerramento

Discussão sobre obesidade

Estabelecimento de metas e objetivos

Psicoeducação sobre o modelo da terapia cognitiva com o "círculo mágico"

Reconhecendo pensamentos automáticos

Apresentação do Registro de pensamentos disfuncionais Perguntas que auxiliam na identificação de pensamentos automáticos disfuncionais

Influência do pensamento sobre os sentimentos e

comportamentos

Discussão sobre o Registro de pensamentos

disfuncionais

Dicas de como obter uma orientação alimentar saudável

Dicas de como trabalhar as desculpas mais comuns para não praticar exercícios físicos

Discussão sobre as orientações alimentares

Revisão do "círculo mágico" e dos registros de

pensamentos disfuncionais

Apresentação das distorções cognitivas - exame e contestação

Discussão sobre o Registro de pensamentos

disfuncionais

Reconhecer as distorções e Mudando a maneira de

pensar

Discussão sobre o registro de pensamentos

disfuncionais

Técnica de como controlar estímulos que levam aos

hábitos alimentares inadequados

Técnica de relaxamento respiração diagragmática

Reconhecimento de emoções e sua relação com a alimentação

Discussão sobre fome, necessidade e saciedade

alimentar

Aplicação da estratégia ACALME-SE

Trabalho com o registro de pensamentos disfuncionais, com as mesmas situações trabalhadas no sétimo encontro

Aplicação do exercício duplo-padrão

Trabalhou-se a técnica construção de alternativas e observação da forma como os outros lidam com as coisas

Revisão das técnicas aplicadas durante o programa para esclarecimento de dúvidas

Solicitação de feedback

Revisadas mais uma vez as técnicas do programa

Revisão das metas e objetivos

Solicitação e retorno de feedback

Encerramento do programa

Coleta de dados
Aplicação de instrumentos

Avaliação do IMC
Registro de pensamentos disfuncionais

Colocar em prática as orientações quanto à alimentação e iniciar a prática de exercícios Identificação das distorções cognitivas mais comuns através dos registros de pensamentos disfuncionais

Registro de pensamentos disfuncionais

Colocar a técnica em prática

Colocar a técnica em prática

Colocar a técnica em prática

Colocar a técnica em prática

Colocar as técnicas em prática 
9) pensamentos ou desejos suicidas; 10) choro; 11) agitação; 12) perda de interesse; 13) indecisão; 14) desvalorização; 15) falta de energia; 16) alterações no padrão de sono; 17) irritabilidade; 18) alterações de apetite; 19) dificuldade de concentração; 20) cansaço ou fadiga e 21) perda de interesse por sexo. ${ }^{36}$

As escalas ESI, ESA e o BDI-II, foram utilizadas apenas para estabelecer medidas de pré e pós teste nos dois grupos de participantes do estudo em relação a interveção da psicologia, para avaliar sua efetividade.

\section{Análise dos dados}

A análise estatística dos dados foi realizada com a utilização do Programa Estatístico Statistical Package for Social Science (SPSS) versão 20.0 (IBM, Chicago, USA). Os testes utilizados na análise estatística do presente estudo através do SPSS foram: Teste de normalidade de Shapiro-Wilk; Comparação intergrupos dados não paramétricos - teste de MannWhitney; dados paramétricos- teste t para amostras independentes; Comparação intragrupos - dados não paramétricos- teste de wilcoxon; dados paramétricos
- teste $t$ de amostras pareadas; Mediana com intervalo interquartílico.

\section{RESULTADOS E DISCUSSÃO}

Não se evidenciou relação de estresse com sobrepeso e obesidade. Em relação aos 21 sintomas depressivos avaliados pela Escala Beck (BDI-II), pode-se observar que o sobrepeso e a obesidade apresentam relação com a capacidade de tomada de decisão e alterações de apetite, que apresentaram nível de significância e concordância entre os grupos avaliados. $\mathrm{O}$ estudo também mostrou efetividade no programa de sessões psicológicas de orientação e treinamento cognitivo, pois seus participantes apresentaram melhora nos sintomas depressivos e na efetiva redução de peso.

Média de Idade inicial: GC- M=13,04 / DP 1,75 anos / GE- M=13,26 / DP 1,39 anos. Sexo: GC 11 masculino e 12 feminino/ GE - 10 masculino e 13 feminino. A Tabela 2 demonstra as médias e desviopadrão de peso, altura e índice de massa corporal dos GE e GC no pré e pós-testes.

Tabela 2 - Médias \pm desvio padrão de peso, altura e índice de massa corporal dos grupos no pré e pós-teste

\begin{tabular}{|c|c|c|c|c|c|c|c|}
\hline & \multicolumn{3}{|c|}{ Experimental } & \multicolumn{3}{|c|}{ Controle } & \multirow{2}{*}{$\mathbf{P}$} \\
\hline & Pré & Pós & $\mathbf{p}$ & Pré & Pós & $\mathbf{p}$ & \\
\hline Peso (kg) & $77,60 \pm 19,09$ & $68,55 \pm 15,90$ & $0,48^{* *}$ & $67,83 \pm 14,92$ & $68,55 \pm 15,90$ & $0,33^{* *}$ & $0,06^{* * *}$ \\
\hline Altura (m) & $1,60 \pm 0,10$ & $1,62 \pm 0,10$ & $0,00^{*}$ & $1,56 \pm 0,09$ & $1,59 \pm 0,08$ & $0,00^{*}$ & $0,11^{* * * *}$ \\
\hline IMC (kg/m²) & $30,19 \pm 7,42$ & $28,97 \pm 7,33$ & $0,03^{* *}$ & $27,74 \pm 4,32$ & $27,11 \pm 4,85$ & $0,02^{* *}$ & $0,28^{* * *}$ \\
\hline
\end{tabular}

${ }^{*}$ Teste de wilcoxon para comparação intragrupo; ${ }^{* *}$ Teste t para amostras pareadas; ${ }^{* * *}$ Teste de Mann-whitney para comparação intergrupos; ${ }^{* * * *}$ Teste t para amostras independentes.

Tabela 3 - Comparação intragrupo no pré e pós-teste do estresse

\begin{tabular}{|c|c|c|c|c|c|c|c|}
\hline \multirow[b]{2}{*}{ *Pré-teste } & \multicolumn{6}{|l|}{ *Pós-teste } & \multirow[b]{2}{*}{$\mathbf{p}^{*}$} \\
\hline & Ausente & Alerta & Resistência & Quase exaustão & Exaustão & Total & \\
\hline \multicolumn{8}{|l|}{ Controle } \\
\hline Ausente & 7 & 0 & 1 & 1 & 1 & 10 & \multirow{6}{*}{0,17} \\
\hline Alerta & 0 & 0 & 0 & 0 & 0 & 0 & \\
\hline Resistência & 0 & 0 & 0 & 0 & 0 & 0 & \\
\hline Quase Exaustão & 2 & 0 & 0 & 0 & 0 & 2 & \\
\hline Exaustão & 10 & 0 & 0 & 1 & 0 & 11 & \\
\hline Total & 19 & $\mathbf{0}$ & 1 & 2 & 1 & 23 & \\
\hline \multicolumn{8}{|l|}{ Experimental } \\
\hline Ausente & 12 & 1 & 0 & 0 & 0 & 13 & \multirow{6}{*}{0,91} \\
\hline Alerta & 0 & 1 & 0 & 0 & 0 & 1 & \\
\hline Resistência & 0 & 0 & 0 & 0 & 0 & 0 & \\
\hline Quase Exaustão & 1 & 1 & 0 & 0 & 0 & 2 & \\
\hline Exaustão & 6 & 1 & 0 & 0 & 0 & 7 & \\
\hline Total & 19 & 4 & $\mathbf{0}$ & $\mathbf{0}$ & $\mathbf{0}$ & 23 & \\
\hline
\end{tabular}

* Teste Wilcoxon

* Os dados do pré teste são apresentados em "itálico" na vertical, na última coluna, e os dados do pós teste são apresentados em "negrito" na horizontal, na última linha.

Rev. Interdisciplin. Promoç. Saúde - RIPS, Santa Cruz do Sul, 1(4):256-266, out/dez. 2018 ISSN: 2595-3664 
Tabela 4 - Comparação intragrupo no pré e pós-teste BDI-II

\begin{tabular}{|c|c|c|c|c|c|c|c|c|}
\hline \multirow[b]{2}{*}{ *Pré-teste } & \multicolumn{5}{|l|}{ *Pós-teste } & \multirow{2}{*}{$\begin{array}{c}\text { Mediana } \\
\text { Pré }^{* *}\end{array}$} & \multirow{2}{*}{$\begin{array}{c}\text { Mediana } \\
\text { Pós }^{* * *}\end{array}$} & \multirow{2}{*}{$\mathbf{p}^{*}$} \\
\hline & Mínimo & Leve & Moderada & Grave & Total & & & \\
\hline \multicolumn{9}{|l|}{ Controle } \\
\hline Mínimo & 14 & 1 & 0 & 0 & 15 & \multirow{5}{*}{9 [4-19] } & \multirow{5}{*}{7 [1-12] } & \multirow{5}{*}{$<0,01$} \\
\hline Leve & 3 & 0 & 0 & 0 & 3 & & & \\
\hline Moderada & 1 & 1 & 0 & 0 & 2 & & & \\
\hline Grave & 0 & 1 & 1 & 1 & 3 & & & \\
\hline Total & 18 & 3 & 1 & 1 & 23 & & & \\
\hline \multicolumn{9}{|c|}{ Experimental } \\
\hline Mínimo & 14 & 0 & 0 & 1 & 15 & \multirow{5}{*}{$8[2-15]$} & \multirow{5}{*}{$2[0-7]$} & \multirow{5}{*}{$<0,01$} \\
\hline Leve & 5 & 0 & 0 & 0 & 5 & & & \\
\hline Moderada & 0 & 0 & 0 & 0 & 0 & & & \\
\hline Grave & 2 & 0 & 1 & 0 & 3 & & & \\
\hline Total & 21 & $\mathbf{0}$ & 1 & 1 & 23 & & & \\
\hline
\end{tabular}

A Tabela 3 demonstra a comparação intragrupo no pré e pós-teste dos instrumentos que avaliaram as fases do estresse, ESI e ESA. Apesar de serem duas escalas, os resultados da classificação são os mesmos como ausente, alerta, resistência, quase-exaustão e exaustão.

A Tabela 4 demonstra os resultados referentes ao BDI-II quanto à intensidade dos sintomas depressivos.

Os resultados do presente estudo podem ser observados conforme os índices de IMC comparados através do teste "t student" entre GC e GE. Para o GE, observa-se uma redução média do IMC de $\mathrm{M}=-1,21$ $\mathrm{kg} / \mathrm{m}^{2} / \mathrm{DP}=2,26$, aproximadamente o dobro quanto ao GC uma redução de $\mathrm{M}=-0,63 \mathrm{~kg} / \mathrm{m}^{2} / \mathrm{DP}=1,42$; em relação ao peso, o GE apresentou redução de $\mathrm{M}=$ $-1,19 \mathrm{~kg} / \mathrm{DP}=4,94$ e o $\mathrm{GC} \mathrm{M}=-0,71 \mathrm{~kg} / \mathrm{DP}=3,56$. Estes dados mostram uma redução significativa no IMC e na perda de peso do GE quando comparados ao GC.

É importante ressaltar que os resultados desta intervenção são de uma equipe interdisciplinar que incluiu educadores físicos, nutricionista e psicólogo. Os tratamentos conjuntos com atividade física, educação nutricional e intervenções cognitivocomportamentais em adolescentes com sobrepeso e obesidade são extremamente benéficos para a diminuição do peso corporal. Além disso, optou-se pela modalidade de grupos, pois sabe-se que a TCC aplicada em grupos de obesos, além da redução efetiva do peso, pode acarretar em benefícios como melhora da autoestima, de sintomas depressivos, redução dos níveis de ansiedade e no bem estar. ${ }^{21,34,39,40}$

Esta modalidade de intervenção é considerada efetiva para perda de peso. O uso da TCC provoca mudanças cognitivas duradouras, partindo do pressuposto que com a alteração do pensamento o comportamento alimentar também será alterado, sendo estas modificações responsáveis por fazerem indivíduos obesos a melhorar a qualidade da sua alimentação e implementar a prática de exercícios físicos. ${ }^{2,34,41,42}$

Os estudos de Taylor, Stonehocker, Steele, Sharma $^{43}$ e Darby, Hay, Mond, Rodgers, Owen ${ }^{44}$ pontuam que, apesar da obesidade não ser uma doença psiquiátrica, esta está associada a transtornos psicológicos como a depressão e geralmente ocasionados por comportamentos alimentares desordenados. Godoy-Matos et al. ${ }^{2}$ também demonstram a relação de sintomas de estresse e depressão com a obesidade, apesar do presente estudo evidenciar não existir correlação significativa entre estresse e obesidade.

Em relação aos 21 sintomas depressivos avaliados pelo instrumento BDI-II, destes, observaram-se de maior incidência nas duas amostras os seguintes sintomas tristeza, sentimentos de culpa, choro, fracasso passado, perda de interesse, indecisão, desvalorização, falta de energia e alterações no apetite; Quando comparados os grupos entre si, foram significativos com maior incidência e ocorrência na amostra total, os sintomas de alterações 
no apetite e indecisão. Em relação à intensidade dos sintomas depressivos constatou-se que dos 23 participantes no GE no pré-teste apresentavam sintomas 5 leve e 3 graves, passando no pós teste para 1 moderado e 1 grave, ou seja, dos oito participantes que apresentavam sintomas depressivos, apenas 2 continuaram com tal sintomatologia. Já, o GC no préteste 3 leve, 2 moderado e 3 grave, passando no pós teste de 3 leve, 1 moderado e 1 grave, ou seja, dos oito que apresentavam sintomas depressivos no pré teste 5 continuaram apresentando sintomas depressivos no pós teste. Analisando os totais do GE de 23 no pré teste 15 estavam com sintomas mínimos e no pós teste 21 no mínimo, já o GC no pré teste 15 no mínimo e após no pós teste 18 no mínimo. Tais dados demonstram a redução da intensidade de sintomas depressivos para o GE da amostra, o que mostra a efetividade da intervenção e a possível correlação de sintomas depressivos com a obesidade.

Estudos ${ }^{45-47}$ demonstram que a dificuldade em manter uma dieta saudável pode estar relacionada a um conflito de objetivos, que seriam o prazer de comer e o controle de peso. A maioria dos obesos que tentam manter uma dieta falha ao se deparar com a alta disponibilidade de alimentos calóricos e, por sua vez, opta pelo prazer ao invés de manter o peso saudável. Por causa de prejuízos de ordem neurobiológica, são mais impulsivos. O que corrobora com os resultados da sintomatologia depressiva, a indecisão e alterações do apetite.

Com os resultados da intervenção psicológica, evidencia-se uma relação significativa de sintomas depressivos com a obesidade e sobrepeso. Também se evidenciou uma redução na intensidade da sintomatologia depressiva no GE comparado ao GC que não participou da intervenção. Não se evidenciou uma possível relação de indicadores de estresse com a obesidade na amostra analisada.

As dificuldades com manutenção de peso e alimentação possuem relação com distorções cognitivas e ciclos de comportamentos disfuncionais. Após o obeso aprender a mudar a forma de pensar sobre sua ingesta, passa a ser capaz de comer com calma, percebendo o que está comendo, apreciando melhor o alimento e persistindo em uma dieta saudável. É possível um aprendizado de novas habilidades cognitivas, mostrado na reestruturação de pensamentos e na redução significativa na frequência das distorções, durante as intervenções no GE. Também é possível observar a aquisição de novas habilidades cognitivas, pois já se observou mudança nos pensamentos, sentimentos e comportamentos, quando comparados os Registros de Pensamentos Diários (RPD) entre as sessões, e a aquisição de novos pensamentos através das técnicas que foram ministradas. É perceptível a gama de novos pensamentos dos participantes em tentar alterar sua cognição em relação às situações trabalhadas nos RPD, no início das intervenções e após ao final. Apesar de alguns pensamentos continuarem dentro das categorias de distorções cognitivas, observa-se a mudança na maneira de pensar dos participantes. Nesse sentido, outros estudos são necessários para ampliar o conhecimento acerca dessas distorções características dessa população.

Os dados demonstram eficiência na intervenção quanto à perda de peso, redução do IMC e a mudança cognitiva. A aplicação em outros contextos ou amostras auxiliaria na ampliação das pesquisas nessa área. Sabe-se que o trabalho com grupos é um campo novo para a TCC, ainda mais com grupo de obesos visando emagrecimento. O presente estudo pode ser reproduzido futuramente com amostras em outras localidades brasileiras para reavaliar a sua efetividade. Como o presente estudo é quantitativo com dados iniciais, os dados e resultados encontrados não podem ser generalizados.

A obesidade é uma condição de difícil controle, com percentuais altos de insucessos terapêuticos e com grande reincidência. Deve-se entender quais fatores estão relacionados à obesidade e que interagem entre si. De forma associada e integrada com outras disciplinas, deve-se favorecer o manejo e controle desta condição que assola grande parte da população mundial. Além disso, é importante promover e capacitar os indivíduos para a aquisição de um repertório qualitativamente maior no combate a esta condição, com respeito à ética e a ciência.

\section{CONCLUSÃO}

Emagrecer implica em mudanças de hábitos de vida e mudanças de comportamento, mas antes disso, mudanças de pensamento. $\mathrm{O}$ presente estudo se refere a um programa de 13 sessões de orientação e treinamento em grupo para escolares com sobrepeso e obesidade, conjuntamente com o trabalho de educadores físicos e nutricionista. Os dados demonstram eficiência na intervenção quanto à perda de peso, redução de sintomas depressivos 
e a mudança cognitiva com aquisição de novas habilidades mentais pelo GE. Tal programa de sessões pode ser reproduzido por profissionais da psicologia para auxiliar como coadjuvante no tratamento da obesidade, pois se mostrou eficaz. O trabalho com grupos é um campo novo para a TCC, ainda mais com grupo de obesos visando emagrecimento. O presente estudo pode ser reproduzido futuramente com amostras em outras localidades brasileiras para reavaliar a sua efetividade.

\section{REFERÊNCIAS}

1. Bertoletti J, Garcia-Santos SC. Avaliação do estresse na obesidade infantil. Psico 2012;43(1):2.

2. Godoy-Matos AF, Oliveira J, Guedes EP, Carraro L, Lopes AC, Mancini MC, Benchimol AK. 2009. Diretrizes brasileiras de obesidade 2009/2010. São Paulo: Ac Farmacêutica; 2009. (Associação Brasileira para o Estudo da Obesidade e da Síndrome Metabólica (ABESO)

3. Rangé BP. (Org.). Psicoterapias cognitivo-comportamentais: um diálogo com a psiquiatria. 2. ed. Porto Alegre: Artmed; 2011. 4. Dalgalarrondo P. Psicopatologia e semiologia dos transtornos mentais. Porto Alegre: Artmed; 2000.

5. Gonçalves S, Silva D, Antunes H. Variáveis psicossociais no excesso de peso e na obesidade infantil. Rev Bras Crescimento e Desenvolv Hum 2012;22(2):179-86.

6. Oliveira A, Albuquerque C, Carvalho GSD, Sendin P, Silva M. Determinantes da obesidade nos adolescentes. Ponta Delgada: Universidade dos Açores; 2009.

7. Sigulem DM, Taddei JAAC, Escrivão MAMS, Devincenzi MU. Obesidade na infância e na adolescência. Compacta Nutr 2001;2:5-16.

8. Luiz AMAG. Efeitos de um programa de intervenção cognitivo-comportamental em grupo para crianças obesas [tese]. São Paulo: Universidade de São Paulo; 2010.

9. Souza JMBD, Castro MMD, Maia EMC, Ribeiro AN, Almondes KMD, Silva NGD. Obesidade e tratamento: desafio comportamental e social. Revista Brasileira de Terapias Cognitivas 2005;1(1):59-67.

10. Vasques F, Martins FC, Azevedo APD. Psychiatric aspects in the treatment of obesity. Rev Psiquiatr Clín 2004:31(4):195-198. doi: http://dx.doi.org/10.1590/S0101-60832004000400013

11. Finger IDR, Potter JR. Entrevista motivacional no tratamento de sobrepeso/obesidade: uma revisão de literatura. Rev Bras Ter Cog 2011;7(2):2-7.

12, Luiz AMAG, Gorayeb R, Liberatore Júnior RDR. Avaliação de depressão, problemas de comportamento e competência social em crianças obesas. Estud Psicol 2010;27(1):41-8. doi: http://dx.doi.org/10.1590/S0103-166X2010000100005

13. Sarmento ASL, Schoen-Ferreira TH, Medeiros ÉH, Cintra IP. Avaliação dos sintomas emocionais e comportamentais em adolescentes obesos. Estud Pesqui Psicol 2010;10(3):833-47.

14. Cataneo C, Carvalho A MP, Galindo EMC. Obesidade e aspectos psicológicos: maturidade emocional, auto-conceito, locus de controle e ansiedade. Psicol Reflex Crít 2005;18(1):3946. doi: http://dx.doi.org/10.1590/S0102-79722005000100006
15. Fitzpatrick KM, Willis D, O’Connor G. Circumstances, resources, and weight status outcomes among middle School Students. The Journal of Early Adolescence 2014;34(8):105874. doi: https://doi.org/10.1177/0272431613518974

16. Adams RE, Cantin S. Self-disclosure in friendships as the moderator of the association between peer victimization and depressive symptoms in overweight adolescents. The Journal of Early Adolescence 2012;33(3):341-62.

17. Schoen-Ferreira TH, Sayegh F, Damaso A, Cintra IP. Relação de amizade de adolescentes obesos e eutróficos. Psicol Rev 2010;16(1):47-63.

18. Fisberg M. Atualização em obesidade na infância e adolescência. São Paulo: Atheneu; 2004.

19, Luiz AMAG, Gorayeb R, Liberatore Júnior RDR, Domingos, NAM. Depressão, ansiedade e competência social em crianças obesas. Estud Psicol 2005;10(1):35-9. doi: http://dx.doi. org/10.1590/S1413-294X2005000300005

20. Neufeld CB. Terapia cognitivo-comportamental em grupo para crianças e adolescentes. Porto Alegre: Artmed Editora; 2015.

21. Neufeld CB, Moreira CAM, Xavier GS. Terapia cognitivocomportamental em grupos de emagrecimento: o relato de uma experiência. Psico 2012:43(1):8.

22. Zimerman DE. Fundamentos básicos das grupoterapias. Porto Alegre: Artmed Editora; 2010.

23. Czeresnia D, Freitas CD. Promoção da saúde: conceitos, reflexões, tendência. Rio de Janeiro: Editora Fiocruz; 2003.

24. Stallard, P. Guia do terapeuta para os bons pensamentos bons sentimentos: utilizando a terapia cognitivo comportamental com crianças e adolescentes. Porto Alegre: Artmed; 2007.

25. Pereira CM, Silva AL. Obesidade e estilos de vida saudáveis: questões relevantes para intervenção. Psic Saúde \& Doenças 2011;12(2):161-82.

26. Apienza G, Schoen T, Fisberg M. Effects of psychological intervention on social competence of obese adolescents. Psic Saúde \& Doenças 2014;15(3):612-22. doi: http://dx.doi. org/10.15309/14psd150305

27. Pereira S. Projeto de intervenção em crianças e adolescentes obesos. O portal dos psicólogos. 2004. Disponível em $<$ http:// www.psicologia.pt/artigos/textos/A0303.pdf $>$. Acesso em $15 \mathrm{de}$ fevereiro de 2019.

28. Wright JH, Basco MR, Thase ME. Aprendendo a terapia cognitivo-comportamental. Porto Alegre: Artmed, 2008.

29. Beck JS. Terapia cognitiva para desafios clínicos: o que fazer quando o básico não funciona. Porto Alegre: Artmed; 2007.

30. Knapp P. Terapia cognitivo-comportamental na prática psiquiátrica. Porto Alegre: Artmed; 2004.

31. Beck JS. Terapia cognitiva: teoria e prática. Porto Alegre: Artmed; 1997.

32. Greenberger D, Padesky CA. A mente vencendo o humor. Porto Alegre: Artmed; 1999.

33. Leahy RL. Técnicas de terapia cognitiva: manual do terapeuta. Porto Alegre: Artmed; 2006.

34. Leahy RL. Livre de ansiedade. Porto Alegre: Artmed; 2011. 35. Beck JS. Pense magro por toda a vida. Porto Alegre: Artmed; 2011.

36. Lipp ME, Lucarelli MD. Escala de stress infantil-ESI: manual. São Paulo: Casa do Psicólogo; 1998.

37. Tricoli VAC, Lipp MEN. Escala de stress para 
adolescentes. São Paulo, Casa do psicólogo; 2005.

38. Gorenstein C, Wang YP, Argimon I L, Werlang BSG. Manual do Inventário de Depressão de Beck-BDI-II. São Paulo: Editora Casa do Psicólogo: 2011.

39. Santana SM, Silva NG, Gonçalves DM, Melo MCMD. 2014. Intervenções em grupo na perspectiva cognitivocomportamental: experiências no contexto da clínicaescola. Rev Bras Ter Cogn 2014;10(1):47-53. doi: http://dx.doi. org/10.5935/1808-5687.20140007

40. Wilfley DE, Kolko RP, Kass AE. Cognitive behavioral therapy for weight management and eating disorders in children and adolescents. Child and Adolescent Psychiatric Clinics of North America 2011:20(2):271-85.

41. Costa MB, Melnik T. 2016. Efetividade de intervenções psicossociais em transtornos alimentares: um panorama das revisões sistemáticas Cochrane. Einstein 2016; 14(2):235-77.

42. Luz FQ, Oliveira MS. Terapia cognitivo-comportamental da obesidade: uma revisão da literatura. Aletheia 2013;(40):159-73. 43. Taylor VH, Stonehocker B, Steele M, Sharma AM. An overview of treatments for obesity in a population with mental illness. Canadian Journal of Psychiatry 2012;57(1):13-20. doi: https://doi.org/10.1177/070674371205700104

44. Darby A, Hay P, Mond J, Rodgers B, Owen C. Disordered eating behaviours and cognitions in young women with obesity: relationship with psychological status. International Journal of Obesity 2007;31(5):876-882.

45. Freitas BI. 2016. Tratamento intensivo baseado na Terapia de Aceitação e Compromisso para dificuldades relacionadas ao peso [Dissertação]. Porto Alegre: Pontifícia Universidade Católica do Rio Grande do Sul; 2016.

46. Fidelix Y, Farias JC, Lofrano-Prado, MC, Guerra, RLF, Cardel M, Prado WL. Intervenção multidisciplinar em adolescentes obesos: preditores de desistência. Einstein 2015 Sept;13(3)38894. doi: https://doi.org/10.1590/S1679-45082015AO3339

47. Stroebe W, Van Koningsbruggen GM, Papies EK, Aarts H. Why most dieters fail but some succedd: a goal conflict model of eating behavior. Psychological Review 2013;120(1):110-38. doi: https://doi.org/10.1037/a0030849

Como citar: LÜDTKE, Lucas et al. Obesidade, depressão e estresse: relato de uma intervenção multidisciplinar em grupo com adolescentes. Revista Interdisciplinar de Promoção da Saúde, Santa Cruz do Sul, v. 1, n. 4, dez. 2018. ISSN 2595-3664. Disponível em: <https://online.unisc.br/seer/index.php/ripsunisc/article/view/13078>. Acesso em: 16 abr. 2019. doi: https://doi. org/10.17058/rips.v1i4.13078. 